\title{
Managing Adaptive Pervasive Computing using Knowledge-based Service Integration and Rule-based Behavior
}

\author{
David Lewis, Owen Conlan, Declan O'Sullivan, Vincent Wade \\ Computer Science Department, Trinity College Dublin, Ireland \\ \{Dave.Lewis, Owen.Conlan,Declan.OSullivan,Vincent.Wade\}@cs.tcd.ie
}

The commonly articulated vision of pervasive computing represents a huge increase in the number of independently developed components that must interoperate and in the level of autonomy they must demonstrate. This motivates a shift to exchanging interoperability knowledge between components at runtime coupled with the ability of components to dynamically adapt themselves to the requirements of users moving between spaces and tasks. The confluence of the service-oriented techniques and ontology-based semantics as Semantic Web Services, offers such dynamic adaptivity through knowledge-based service composition.

We aim to establish a conceptual architecture for pervasive computing that integrates semantic service composition and policy-based management in providing collective behavior that adapts to the user's changing needs, but which conforms to the goals of those responsible for the resources used by those services. This architecture must remain simple enough to have the potential for ubiquitous deployment over restricted computing resources and be flexible enough to accommodate the rollout of new automated reasoning techniques. The core feature of our architecture is the Adaptive Service Element (ASE), which is characterized by a service description, a model of the state observable by the ASE, a description of the services it makes use of and a rule-based model for describing and restricting its behavior. The lifecycle of an ASE is managed primarily through the bindings made between these models. Service Descriptions are specified in OWL-S language (http://www.daml.org/services) where a service is described using a description logic ontology specifying inputs, outputs, preconditions and effects. Compatible with the OWL-S service description, the ASE state view is an ontological model of the objects of which the element is aware, including managed resources, external context and operational state such as counters and timers. An ASE's behavior rules are in the form event-conditions-actions and dictate the behavior of the service in reacting to: the service's invocation, the access control policies of the service provider, the resource management policies of the resource owner and changes to state objects. Meta-rules, typically established by the ASE developer, restrict how available events, conditions and actions can be constructed into behavior rules, thus restricting unwanted rule behavior. ASEs may be generated by automated service composers and thus have entirely rule based 
behavior, or they may be pre-implemented software components with restricted rule-based behavior for flexibly enforcing policies to invoke external services, such as accounting or fault management, when specific events occur.

This architecture provides us with a basis for investigating knowledge-based approaches to the development, deployment and management of services and service compositions. We are exploiting an ontological model of context information to link natural language task descriptions to OWL-S based service requests generated by Bayesian network-based inference [1]. However, we recognize that real world context involves wide ontological heterogeneity, so we are developing a universal context querying service for intelligently distributing queries over disparate context knowledge sources, initially by exploiting the ontology merging properties of Topic Maps [3]. Given a high level semantic service task definition, we are extending an existing multi-model adaptive hypermedia engine, for use as an automated service composer. This resolves the service request, OWL-S models of available ASEs and service composition patterns and relevant context information, such as the user's terminal capabilities. Both the service composition process and the querying of heterogeneous context knowledge sources present problems of semantic interoperability between differing ontologies, and here we are examining the limitation of applying existing ontologymerging algorithms to the runtime generation of semantic gateways [2]. The resulting composite service ASE will possess behavior rules that control how it invokes other ASEs' services. Any operational policies applied to such a composite service, must be mapped to suitable behavioral rules for the constituent ASEs. Such policy mapping problems remain problematic, however we are investigating whether the ontological links between a composite service, its constituent services and the relevant ASE state models, will provide sufficient reasoning context to generate low level policies automatically. Overall we observe that the potential to use the same rule based mechanisms for controlling the execution of control and data flow in service compositions (both centralized and decentralized) and for enforcing rule based management policies points to a rules based inference service, possibly based on RuleML, as a key infrastructural requirement for an ontologydriven adaptive service platform. Such an integrated approach underlines the lack of a real distinction between service control and service management in the highly adaptive systems that will be required for pervasive computing environments.

[1] Higel S., O'Donnell T., Lewis D., Wade V. "Towards an Intuitive Interface for Tailored Service Compositions", Proc. Of Distributed Applications and Interoperable Systems, Paris, Nov 2003, LNCS 2893, pp. 266-273

[2] O’Sullivan, D., Lewis, D., "Semantically Driven Service Interoperability for Pervasive Computing", Proceedings of the 3rd ACM International Workshop on Data Engineering for Wireless and Mobile Access, San Diego, Sept. 2003, pp 17-24.

[3] Power, R., "Topic Maps for Context Management", Proceedings of the International Symposium on Information and Communication Technology, Dublin, Sept 2003, pp 207-212.

This work is funded by the Irish Higher Education Authority under the M-Zones programme. 